The BMJ

Cite this as: BMJ 2021;375:n3014 http://dx.doi.org/10.1136/bmj.n3014 Published: 03 December 2021

\title{
Can you do what l'm asking you to do?
}

\section{Tessa Richards discusses new approaches to tackling health and digital divides, including two questions that clinicians should ask their patients at the end of each consultation}

\section{Tessa Richards senior editor patient partnership}

The rapid move to remote healthcare services, triggered by the covid-19 pandemic, is stimulating debate on their impact on existing health divides. As new methods of care are adopted how do we avoid baking in further inequities, asked Mary Dixon-Woods, director of the THIS Institute, as she opened the institute's recent webinar on the burden of treatment and remote care.

An instant response from Frances Mair, head of general practice and research convenor for the Institute of Health and Wellbeing at the University of Glasgow, was abandon the "one size fits all" approach to service provision. Health systems must acknowledge, analyse, and reduce the "structurally induced" burdens they impose on patients, she said, which reduce their ability to adhere to treatment. ${ }^{1}$ Services must be tailored to take account of people's circumstances, needs, abilities, and preferences. ${ }^{2}$

The burden of navigating health services is particularly heavy for those who live with multi morbidity, which Mair described as health systems "biggest global problem this century." This is not just a challenge for older people. Rates of multimorbidity are disproportionately high in low socioeconomic groups where the development of multiple conditions occurs 10 years earlier than in richer populations. Health systems need to look at and learn from patients' experience of accessing healthcare, interacting with health professionals, and the extent to which they can and can't follow advice and self manage conditions.

Patients' capacity to cope with complex management regimens and new technologies is influenced by many factors including environmental conditions, work and family responsibilities, support networks, cognitive ability, and personal skills. Knowledge of these factors is key to developing good quality, person centred services which are "simpler, kinder and less disruptive" than the ones currently being provided. While this knowledge takes time to glean a simple measure clinicians can adopt, said Mair, is to ask patients two questions at the end of a consultation:

Do you think what I am asking you to do is right for you?

If so, can you do it?

Remote care services have reduced the time, effort, and cost to patients of travelling to attend hospital and GP appointments and recent studies show most patients (and many staff) have welcomed them. But there are caveats about quality of care and it is evident that there is much still to be learnt by patients and healthcare professionals alike on how to make remote care services and consultations work well. ${ }^{34}$
But remote care has not worked for all, and has been easier to embed in some specialties than others. Those who lack access to digital technologies or the skills to use them to the best advantage have faced problems. And even the best forms of remote care can't fully compensate for the valuable non verbal information conveyed during face to face consultations, and the opportunity they provide to express empathy and forge strong doctor-patient relationships. Video consults are widely seen as "next best" to face to face interactions, but are offered less often than teleconsults and both raise concerns about lack of privacy and confidentiality.

My experience of remote care in the era of covid has been one of diktat by smartphone messages to which one cannot reply and no choice about the form of consultation. On occasions I have been frustrated (unreasonably no doubt) by teleconsultations with new doctors who have not got to grips with my ongoing medical problems.

But I shouldn't complain for it's not easy for doctors either, and I am at least health literate and have the technological skills needed to cope with remote care. Many don't, and in our brave new world of remote care and sophisticated digital monitoring, raising health literacy and the digital skills of those who lack them is key to tackling health divides.

A recent review of health literacy as a social determinant of health underlines that people with low literacy levels experience worse outcomes for they are less likely to use disease prevention services and less able to self manage chronic conditions. ${ }^{5}$ Exploring ways to raise literacy levels was discussed at a second webinar last week held by The BMJ where debate centred on how to avoid real time access to full personal electronic health records which is a growing global movement exacerbating health inequalities.

Amir Hannan, a UK GP, who has been providing patients with access to their EHRs for 16 years has taken a hands on personal approach to support his patients to access, learn from, and use their own health information. In the US, The Los Angeles County Health Service has provided patients with access to their medical records online via a patient portal since 2015. Around a third of patients regularly read their notes, said Anshu Abhat, director of digital health equity. But those in lower socio economic groups, older people, and ethnic minorities are less likely to do so, and that has spurred the development of a health technology navigator programme to support them. A similar programme to tackle literacy divides using trained community digital health mentors has been funded by the Australian Digital 
Health Agency as part of its ambitious plans to transform healthcare through digital technologies. ${ }^{6}$

Debate about post covid service provision tends to focus on people's access to technology and the internet rather than exploring what methods of communication work best in diverse communities of patients. But evidence suggests that the greatest barriers to adopting digital health tools are not technical, but related to facilitating individual, organisational, and system change. ${ }^{7}$

Not before time, it is now acknowledged that health literacy is "not solely reliant on individual capabilities, but also on organisations' ability to make health related information and services equitably accessible and comprehensible." ${ }^{8}$ The message could hardly be clearer. High standards of patient and public involvement and collaborative co-design of services are key to avoiding new models of care widening existing health divides.

Competing interests: none declared.

Provenance and peer review: Commissioned, not peer reviewed

1 Gallacher KI, May CR, Langhorne P, Mair FS. A conceptual model of treatment burden and patient capacity in stroke. BMC Fam Pract2018;19:9. doi: 10.1186/s12875-017-0691-4. pmid: 29316892

2 Mair FS, Montori VM, May CR. Digital transformation could increase the burden of treatment on patients. BM/2021;375:n2909. doi: 10.1136/bmj.n2909. pmid: 34824093

3 Providers NHS. What patients and staff really think about remote consultations.

https://nhsproviders.org/news-blogs/blogs/what-patients-and-staff-really-think-about-remoteconsultations

4 National Voices. Dr will zoom you now. https://www.nationalvoices.org.uk/publications/ourpublications/dr-will-zoom-you-now-getting-most-out-virtual-health-and-care

5 Nutbeam D, Lloyd JE. Understanding and Responding to Health Literacy as a Social Determinant of Health. Annu Rev Public Health 2021;42:159-73.

doi: 10.1146/annurev-publhealth-090419-102529. pmid: 33035427

6 https://www.digitalhealth.gov.au/newsroom/media-releases/recent-media-releases/good-thingshappening-to-support-digital-health-literacy

7 Use of digital health tools in Europe: before, during and after COVID-19 https://eurohealthobservatory.who.int/publications/i/use-of-digital-health-tools-in-europe-before-during-and-after-covid19

8 Santana S, Brach C, Harris L, etal. Updating Health Literacy for Healthy People 2030: Defining Its Importance for a New Decade in Public Health. J Public Health Manag Pract 2021;27(Suppl 6):S258-64. doi: 10.1097/PHH.0000000000001324. pmid: 33729194 